\title{
Registros De Representação Semiótica E O Conceito De \\ Inequação: Análise Do Desempenho De Licenciandos Em \\ Matemática À Luz Da Congruência Semântica
}

\author{
Registers Of Semiotic Representation And The Concept Of Inequality: \\ Analysis Of The Performance Of Mathematics Students In The Light Of \\ Semantic Congruence
}

\author{
Wilian Barbosa Travassos* \\ Universidade Estadual de Maringá - (UEM) \\ Marcelo Carlos de Proença** \\ Universidade Estadual de Maringá - (UEM)
}

\begin{abstract}
Resumo
Neste artigo, o objetivo foi analisar o desempenho de estudantes de um curso de Licenciatura em Matemática em atividades envolvendo o conceito de inequação do $1^{\circ}$ grau com uma variável nos diferentes níveis de congruência semântica. A pesquisa desenvolvida teve como sujeitos 16 acadêmicos de um curso de Licenciatura em Matemática de uma Universidade pública do Estado do Paraná. Para coleta e análise dos dados, foram elaborados oito exercícios sobre o conceito de inequação do $1^{\circ}$ grau com uma variável de modo que fosse aumentando o grau de dificuldade, com base nos três critérios de congruência semântica. Os resultados aqui apresentados evidenciam dificuldades dos acadêmicos especialmente referentes a exercícios cujos critérios de univocidade semântica terminal e correspondência semântica não são satisfeitos, contudo, apresentam também erros envolvendo operações de tratamento, tais como multiplicação, divisão, e propriedades do conceito de inequação. De modo geral, este estudo identificou um baixo desempenho dos acadêmicos neste instrumento de pesquisa, inclusive estudantes dos anos finais da graduação.
\end{abstract}

Palavras-chave: Álgebra; Inequações; Registros de Representação Semiótica

\begin{abstract}
In this article, the objective was to analyze the performance of students of a Mathematics course in activities involving the concept of 1 st degree inequality with a variable in the different levels of semantic congruence. The research developed had 16 students of an undergraduate degree in Mathematics from a public University of the State of Paraná. For the collection and analysis of the resolutions, were elaborated eight exercises on the concept of first-degree inequality with a variable increasing in increasing order the degree of difficulty based on the three conditions of semantic congruence. The results presented here evidenced difficulties of academics especially in the exercises that the conditions of terminal semantic unicity and semantic correspondence are not satisfied, however, they also present errors involving treatment operations, such as multiplication, division, and properties

\footnotetext{
* Mestre em Educação para a Ciência e a Matemática (UEM). Estudante de doutorado pelo Programa de Pósgraduação em Educação para a Ciência e a Matemática (UEM), Maringá, Paraná, Brasil. E-mail: wiliantravassos@hotmail.com

** Doutor em Educação para a Ciência (UNESP). Professor adjunto do Departamento de Matemática (UEM), Maringá, Paraná, Brasil. E-mail: mcproenca@uem.br
} 
of the concept of inequality. The general results of this study presented a low performance of the students in this research instrument, including students of the final years of graduation.

Keywords: Algebra; Inequalities; Semiotic Representation Registers

\section{Introdução}

Apresentamos neste artigo parte dos resultados de uma pesquisa de mestrado voltada a análise do desempenho de estudantes de graduação em Licenciatura em Matemática em atividades envolvendo o conceito de inequação e suas diferentes representações. Deste modo, este artigo focaliza-se nas análises e discussões realizadas referentes a um dos instrumentos de pesquisa da dissertação.

No que se refere ao conceito de inequação, objeto matemático de estudo do presente artigo, segundo alguns documentos curriculares que regem a educação no Brasil tais como os Parâmetros Curriculares Nacionais - PCN (BRASIL, 1998) e a Base Nacional Comum Curricular - BNCC (BRASIL, 2017), o conteúdo de inequações é inicialmente trabalhado no Ensino Fundamental, especialmente nos anos finais, e aprofundado seu conteúdo no Ensino Médio (BRASIL, 2000).

Nesse sentido, o trabalho envolvendo o conteúdo inequações faz parte da formação do professor de Matemática. Desse modo, realizamos buscas em diferentes bancos de dados, tais como Google Acadêmico $^{1}$; Biblioteca Digital Brasileira de Teses e Dissertações (BDTD) ${ }^{2}$ do Instituto Brasileiro de Informação em Ciência e Tecnologia (IBICT) e no Banco de Teses e Dissertações da CAPES $^{3}$ (Coordenação de Aperfeiçoamento de Pessoal de Nível Superior) buscando pesquisas referentes as dificuldades com inequações do $1^{\circ}$ grau, em especial, relacionadas aos estudantes da Licenciatura em Matemática.

Deste modo, encontramos cinco pesquisas: Melo (2007); Campos e Giusti (2008); Souza (2008); Magalhães (2013) e Travassos e Rezende (2017). A dissertação de Melo (2007) teve como objetivo detectar "como professores de um curso de Licenciatura em Matemática desenvolvem desigualdades e inequações com suas classes e quais as fontes orientadoras de seu trabalho a respeito desses assuntos" (MELO, 2007, p.4). Os sujeitos da pesquisa foram quatro professores que lecionam em pelo menos uma disciplina do $1^{\circ}$ ano do curso de Licenciatura Plena em Matemática de uma Universidade do Estado de São Paulo.

\footnotetext{
${ }^{1}$ https://scholar.google.com.br

2 http://bdtd.ibict.br

${ }^{3}$ http://bancodeteses.capes.gov.br
} 
O trabalho de Campos e Giusti (2008) teve como objetivo "identificar os aspectos formais intuitivos e algorítmicos presentes na resolução de desigualdades algébricas com uma incógnita real" (CAMPOS; GIUSTI, 2008, p. 40), cuja pesquisa teve como sujeitos 21 alunos do primeiro ano de um curso de Licenciatura em Matemática.

A tese de Souza (2008) teve como objetivo verificar se uma abordagem envolvendo o tratamento e a conversão de registros pode desencadear uma discussão global a respeito de sua resolução, no que se refere as equações e/ou inequações. Os sujeitos da pesquisa foram dois grupos, sendo um composto por 30 estudantes do primeiro ano de um curso de Licenciatura em Matemática e um grupo de 10 professores da formação continuada.

A pesquisa de Magalhães (2013), teve como objetivo analisar qual seria o impacto de um trabalho envolvendo uma sequência de atividades de sala de aula, voltadas ao desenvolvimento do pensamento algébrico e funcional, realizada por alunos do curso de matemática. Os sujeitos da pesquisa foram 29 alunos do curso de licenciatura em Matemática de uma instituição pública do Estado de Minas Gerais. Apesar de não especificar de quais anos acadêmicos os sujeitos são, a pesquisa dá indícios de que sejam dos primeiros anos do curso.

A pesquisa de Travassos e Rezende (2017), teve como objetivo analisar o desempenho aritmético e algébrico de estudantes do primeiro ano de um curso de Licenciatura em Matemática ao trabalhar com o tratamento de inequações.

Verificamos que tais estudos buscaram analisar resultados sobre conhecimentos de estudantes de licenciatura em Matemática do primeiro ano do curso. Além disso, nenhum desses estudos apresentam análise do desempenho desses estudantes em atividades envolvendo o conceito de inequação em um ponto importante da teoria dos Registros de Representação Semiótica de Duval, a saber: níveis de congruência semântica.

Deste modo, tivemos como objetivo neste artigo o de analisar o desempenho de estudantes dos quatro anos de um curso de Licenciatura em Matemática em atividades envolvendo o conceito de inequação do $1^{\circ}$ grau com uma variável nos diferentes níveis de congruência semântica.

\section{Teoria Dos Registros De Representação Semiótica}

A Teoria dos Registros de Representação Semiótica é oriunda de estudos relacionados a psicologia cognitiva de modo a contribuir com a aprendizagem em matemática. A aprendizagem matemática por sua vez apresenta algumas peculiaridades relacionadas a sua compreensão 
devido a abstração que a mesma possui, o que implica na utilização de sistemas de representações que possibilitem o acesso a seus objetos matemáticos (DUVAL, 2009).

Deste modo, Duval (2009) menciona que: “[...] não se pode ter compreensão em matemática, se nós não distinguimos um objeto de sua representação" (2009, p. 14).

No entanto, eis que surge uma indagação: como diferenciar um objeto matemático de sua representação? Em razão de que os objetos matemáticos só são acessíveis por meio de representações. Esse conflito de reflexões gerou o que Duval define por "paradoxo cognitivo da matemática" (DUVAL et al, 2013, p. 17).

É primordial distinguir objeto matemático de suas representações, uma vez que esta distinção faz parte do processo de compreensão do objeto matemático a nível conceitual. As inequações, por exemplo, podem ser representadas por meio de um intervalo na reta geométrica, um intervalo numérico, uma expressão algébrica, dados dispersos em registro tabular ou até mesmo em língua natural. São essas diferentes representações do conceito matemático inequação que o torna possível de ser identificado.

No tocante, nota-se que um único conceito matemático pode assumir várias representações, variando de acordo com suas propriedades e definições. Deste modo, para designar os diferentes tipos de representações semióticas que um conceito matemático pode assumir, Duval (2003) utiliza-se do termo "registros" de representação de Descartes, fazendo uma releitura do mesmo, classificando assim quatro grandes tipos de registros de representação distintos entre si, conforme apresenta-se no quadro 1 a seguir.

\begin{tabular}{|c|c|c|}
\hline & REPRESENTAÇÃO DISCURSIVA & REPRESENTAÇÃO NÃO DISCURSIVA \\
\hline $\begin{array}{l}\text { REGISTROS } \\
\text { MULTIFUNCIONAIS: } \\
\text { Os tratamentos não são } \\
\text { algoritmizáveis }\end{array}$ & $\begin{array}{l}\text { Língua natural } \\
\text { Associações verbais (conceituais). } \\
\text { Forma de raciocinar: } \\
\text { • Argumentação a partir de } \\
\text { observações, de crenças...; } \\
\text { - Dedução válida a partir de } \\
\text { definçãa ou de teoremas. }\end{array}$ & $\begin{array}{l}\text { Figuras geométricas planas ou em } \\
\text { perspectivas (configurações em dimensão } \\
0,1,2 \text { ou } 3 \text { ). } \\
\text { - Apreensão operatória e não somente } \\
\text { perceptiva; } \\
\text { - Construção com instrumentos. }\end{array}$ \\
\hline $\begin{array}{l}\text { REGISTROS } \\
\text { MONOFUNCIONAIS: } \\
\text { Os tratamentos são } \\
\text { principalmente } \\
\text { algoritmos. }\end{array}$ & $\begin{array}{l}\text { Sistemas de escritas: } \\
\text { - Numéricas (binária, decimal, } \\
\text { fracionaria...); } \\
\text { - Algébricas; } \\
\text { - Simbólicas (línguas formais). } \\
\text { Cálculo }\end{array}$ & $\begin{array}{l}\text { Gráficos cartesianos. } \\
\text { - Mudanças de sistema de coordenadas; } \\
\text { - Interpolação, extrapolação. }\end{array}$ \\
\hline
\end{tabular}

Quadro 1 - Classificação dos diferentes registros mobilizáveis no funcionamento matemático (fazer matemático, atividade matemática)

Fonte: Duval (2003, p. 14).

Observando o quadro 1, nota-se a variedade de representações que um registro possui. Peguemos como exemplo o registro maior sistemas de escrita, na qual é possível classificar as representações desse registro em Numéricas do tipo binária, decimal, fracionaria etc., 
algébricas; simbólicas, etc. Ou seja, há um leque de possibilidades de representações semióticas que um objeto matemático pode assumir, podendo uns terem mais, outros menos.

Observe que até aqui elencamos alguns fatores importantes no processo de compreensão de um conceito matemática, pautados na Teoria dos Registros de Representação Semiótica. A partir daqui, afunilamos a apresentação da teoria aos procedimentos essenciais necessários bem como alguns dos fatores que podem influenciar no processo de compreensão conceitual de um objeto matemático.

Duval (2003) conjectura que "a compreensão em matemática supõe a coordenação de ao menos dois registros de representações semióticas" (2003, p. 15). Neste sentido, coordenar dois registros tem como consequência a "transição" entre um e outro registro. É estabelecer conexões entre os registros de modo que possa trabalhar com o objeto matemático em suas representações distintas, obedecendo as propriedades e definições inerentes a cada registro e reconhecendo-os em suas diferentes formas.

Para que a coordenação de registros ocorra, alguns processos transformadores das representações semióticas são primordiais. Processos estes denominados tratamento e conversão (DUVAL, 2003, 2009).

"Um tratamento é a transformação de uma representação obtida como dado inicial em uma representação considerada como terminal em relação a uma questão, a um problema ou a uma necessidade, os quais fornecem o critério de parada na série de transformações efetuadas" (DUVAL, 2009, p. 57). De modo conciso, os tratamentos são as transformações que ocorrem internamente a um sistema ou registro de representação semiótica.

Referente ao tratamento, alguns exemplos podem ser destacados, tais como a paráfrase, que reformula ou explica um enunciado presente em língua natural ou por exemplo, o cálculo algébrico, que substitui e/ou realiza modificações na escritura de números de modo que não altere o registro inicial a qual representa o conceito.

O quadro 2 a seguir apresenta um exemplo de tratamento referente ao cálculo algébrico em uma inequação do $1^{\circ}$ grau com uma variável. 


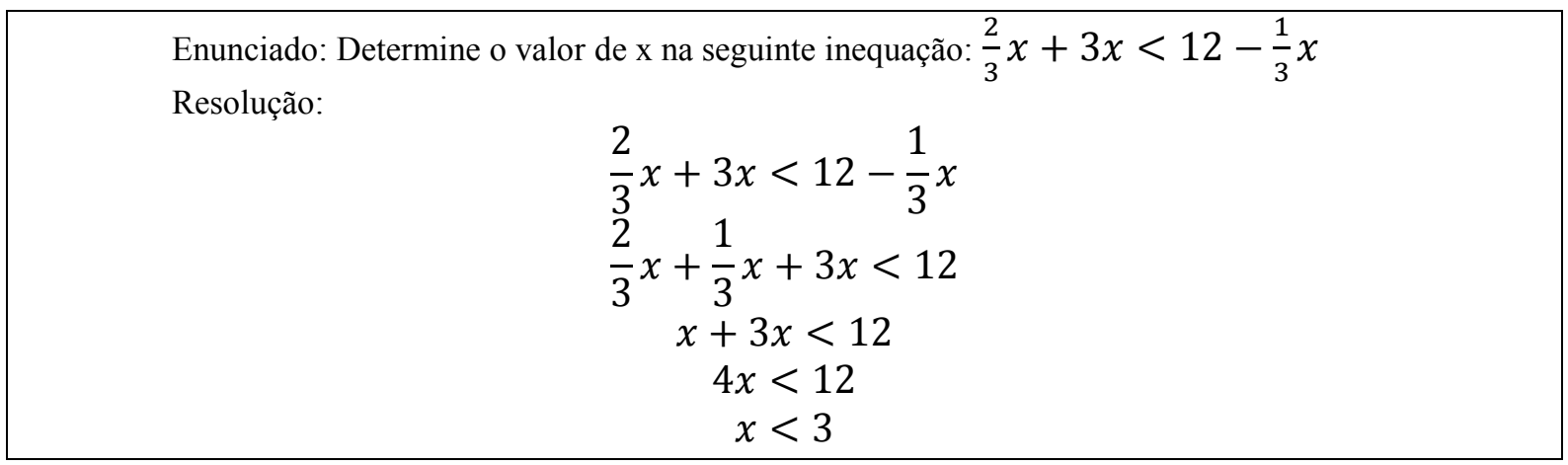

Quadro 2 - Exemplo de tratamento algébrico em uma inequação do $1^{\circ}$ grau com uma variável. Fonte: autores.

Na resolução apresentada no quadro 2, note que as transformações realizadas no registro inicial (inequação algébrica fornecida no enunciado do exercício) são estritamente referentes ao registro algébrico, não alterando o registro de representação no processo de tratamento, nem na solução final.

Já para a conversão: “converter é transformar a representação de um objeto, de uma situação ou de uma informação dada num registro em uma representação desse mesmo objeto, dessa mesma situação ou da mesma informação num outro registro" (DUVAL, 2009, p. 58). De modo conciso, a conversão ocorre quando há transformações externas ao registro inicial, ocorrendo assim mudança de registro.

Da mesma forma que o tratamento, algumas operações podem ser consideradas como um processo de conversão de registros, como por exemplo: a ilustração, na qual consiste na correspondência de uma frase, uma palavra ou até mesmo um enunciado com uma figura ou com algum de seus elementos. Outro exemplo é o processo inverso, ou seja, de uma figura para um texto, que neste caso pode ser considerado como uma descrição ou interpretação (DUVAL, 2009).

O quadro 3 a seguir apresenta um exemplo envolvendo a conversão de registros atrelado ao conceito de inequação.

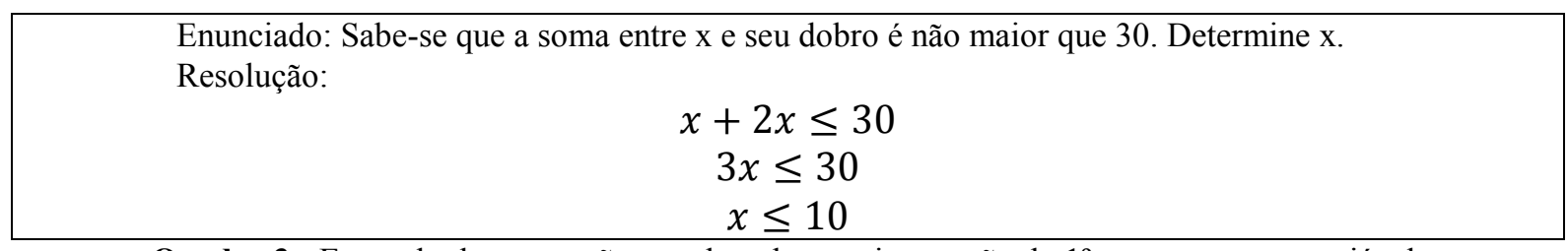

Quadro 3 - Exemplo de conversão envolvendo uma inequação do $1^{\circ}$ grau com uma variável. Fonte: autores.

Observando o quadro 3, note que para encontrar o valor de $\mathrm{x}$, foi necessário montar uma expressão matemática com base nos dados fornecidos em língua corrente no enunciado, ou seja, neste caso realizou-se uma conversão do registro língua natural para o registro sistemas de 
escrita - algébrico. É este processo de troca de registros denominado conversão.

Contudo, o processo de conversão pode enfrentar algumas condições que tornam o processo de transformações de registros mais complexo ou mais fácil, variando de acordo com especificidades de cada registro bem como a maneira como é organizado e apresentado, condições estas resultadas do fenômeno definido por Duval (2009) de não-congruência.

Para determinar se duas representações são congruentes ou não, é preciso começar por segmenta-las em suas unidades significantes respectivas, de tal maneira que elas possam ser colocadas em correspondência. Ao final dessa segmentação comparativa, pode-se então ver se as unidades significantes são, em cada um dos dois registros, unidades significantes simples ou combinações de unidades simples. (DUVAL, 2009, p. 66).

Este tipo de análise permite precisar três critérios de congruência semântica segundo Duval (2009). São eles:

- Correspondência semântica dos elementos significantes: a cada unidade significante simples no registro de partida, corresponda a uma unidade significante elementar no registro de chegada.

- Univocidade semântica terminal: para cada unidade significante no registro de partida, corresponda a apenas uma só unidade significante elementar no registro de chegada.

- Organização das unidades significantes: refere-se a ordem de "incidência" que a organização das unidades das duas representações correspondem.

Neste caso, como estamos trabalhando com o registro de partida língua natural, considera-se como unidade significante os termos que compõe o grupo lexical e semântico do conceito de inequação, ou que possa ser associado ao conceito e/ou sua representação terminal de alguma forma.

Tomemos como exemplo a conversão apresentada no quadro 3, a qual exemplificou a conversão do registro em língua natural para a representação nas escrituras algébricas, para analisarmos sua congruência perante os três critérios de congruência semântica supracitados.



Figura 1 - Exemplo de conversão não congruente do registro língua natural para as escrituras algébricas Fonte: autores.

Sublinhado com um traço azul, representam-se as unidades significantes que compõe o enunciado, juntamente com um círculo preto abaixo com uma letra em seu interior para designar 
cada unidade significante.

No que se refere a correspondência semântica ( $1^{\circ}$ critério), observe que o exercício apresenta correspondência semântica entre as unidades significantes, uma vez que para cada unidade significante em uma das representações, é possível associá-la a uma unidade significante na outra representação.

Para o critério de univocidade semântica terminal ( $2^{\circ}$ critério), nota-se que o exercício não contempla tal condição, visto que, a unidade $D$ do registro de chegada (escrituras algébricas) possui um só valor significante, enquanto que o registro de partida (língua natural) possui duas unidades significantes elementares possíveis de serem representadas no registro terminal (algébrico), por exemplo = (igual) e/ou $<$ (menor). Neste caso a univocidade semântica terminal não é satisfeita.

Para a ordem das unidades significantes ( $3^{\circ}$ critério), nota-se que tal critério também não é satisfeito, pois, no registro de partida, a unidade $B$ (soma) deveria vir após a unidade $A$ (x) para que a ordem das unidades significantes fossem satisfeitas.

Quando o estudante tem conhecimento destes termos e consegue identificá-los como unidades significantes do conceito de inequação, a conversão se torna um processo mais simples de realizar. Contudo, quando o estudante não tem familiaridade com tais termos, ou ainda, sua compreensão a respeito do conceito de inequação é parcial, situações como estas apresentadas neste artigo podem facilmente serem interpretadas erroneamente, sobretudo, confundidas com o conceito de equação

Contudo, pode-se alterar parcialmente ou totalmente o nível de congruência desta atividade por meio de um tratamento no registro de partida (enunciado em língua natural) tornando a conversão um simples processo de codificação.

A figura 2 a seguir exemplifica o exposto.

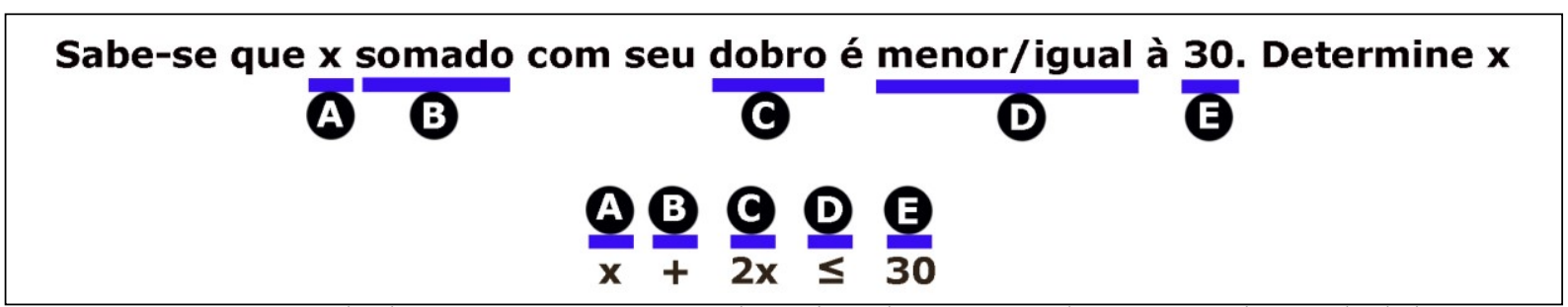

Figura 2 - Exemplo de conversão congruente do registro língua natural para as escrituras algébricas. Fonte: autores.

Observe que neste exemplo, devido ao tratamento realizado no registro de partida em língua natural, reformulou-se as unidades significantes bem como sua organização de modo que deixasse a atividade de conversão congruente, conforme os três critérios de congruência semântica. 
Esses critérios permitem determinar a congruências entre duas representações semioticamente diferentes e representando, ao menos, parcialmente o mesmo conteúdo. Duas representações são congruentes quando há correspondência semântica entre suas unidades significantes, univocidade semântica terminal e mesma ordem possível de apreensão dessas unidades nas duas representações. Naturalmente, pode não haver correspondência para nenhum desses critérios, para dois ou somente para um. A não-congruência entre duas representações pode então ser maior ou menor. A dificuldade da conversão de uma representação depende do grau de não-congruência entre a representação de partida e a representação de chegada (DUVAL, 2009, p. 69).

Dessa forma, pode-se afirmar que, uma atividade congruente tende a ser mais fácil sua resolução, por outro lado, na medida em os critérios de congruência semântica não são satisfeitos, a conversão deixa de ser um processo simples e se torna uma atividade complexa de conversão, exigindo uma atividade cognitiva maior para sua realização. Contudo, pode-se inferir que na medida em que o estudante realiza corretamente a conversão, mesmo estando em níveis altos de não-congruência, é um forte indício de que o estudante possa realmente ter compreendido o objeto matemático a nível conceitual.

\section{Metodologia}

O presente estudo é de natureza quanti-qualitativa, voltado a modalidade de pesquisa exploratória. Segundo Gil (2008) as pesquisas exploratórias proporcionam uma visão holística de determinado tema que geralmente é pouco explorado e, consequentemente, difíceis de se formular hipóteses precisas.

Deste modo, para atender as necessidades do estudo, abordou-se como sujeitos de pesquisa quatro acadêmicos de cada ano $\left(1^{\circ}, 2^{\circ}, 3^{\circ}\right.$ e $4^{\circ}$ anos $)$ de um curso de Licenciatura em Matemática, cujo total foram 16 acadêmicos.

Para o desenvolvimento da pesquisa, elaboramos um instrumento composto por oito exercícios em língua natural de modo que atendesse aos quatro níveis possíveis de congruência semântica, aqui denominados como: nível de congruência 3, atendendo assim aos três critérios de congruência semântica; nível de congruência 2, atendendo a dois critérios de congruência; nível de congruência 1 , no qual a atividade apresenta apenas um dos três critérios de congruência semântica e, o nível não-congruente, no qual este último não atende a nenhum dos critérios de congruência semântica.

O quadro 4 a seguir apresenta o instrumento diagnóstico dessa pesquisa no qual comtempla os oito exercícios envolvendo congruência semântica. 
1) Determine $x$ sabendo que seu valor somado com 2 vezes o seu próprio valor é maior que 27.

2) Pensei em um número $x$, em seguida mulpliquei-o por -3 de modo que seu valor fosse um número maior que 14. Que número pensei inicialmente?

3) Não é igual, nem maior que 12 a diferença entre x e seu triplo. Represente algebricamente o enunciado, em seguida, resolva utilizando os procedimentos necessários.

4) Um número inteiro somado com seu igual não é menor que 2 multiplicado com o seu triplo. Represente algebricamente o enunciado, em seguida, resolva utilizando os procedimentos necessários.

5) É no mínimo 17 a soma entre um número par e seu sucessor. Quais valores esse número pode assumir de modo que essa relação seja satisfeita?

6) Determine $x$ de modo que ao realizar a diferença com seu dobro resulte sempre em um número não negativo.

7) Sabe-se que o valor máximo que x pode assumir é igual o produto entre 3 e seu dobro. Determine $\mathrm{x}$.

8) Sabe-se que o limite inferior dos valores que um número x somado com 8 pode assumir é igual a diferença de dois números iguais. Determine $\mathrm{x}$.

\section{Quadro 4 - Instrumento diagnóstico.}

Fonte: autores.

Os exercícios 1 e 2 foram elaborados de maneira que atendessem as três condições de congruência semântica, ou seja, são exercícios congruentes. Os exercícios 3 e 4 foram elaborados apresentando em sua constituição dois critérios de congruência semântica, no qual o exercício três não atende ao critério 3 , sendo este relacionado a organização das unidades significantes, e o exercício 4 não atende ao critério 2 , sendo este relacionado a univocidades semântica terminal.

Os exercícios 5 e 6 apresentam em seu enunciado condições que atendem a apenas um dos critérios de congruência semântica. $\mathrm{O}$ exercício 5 satisfaz somente o critério 1 , sendo este relacionado a correspondência das unidades significantes. Já o exercício 6 satisfaz apenas o critério 3, relacionado a organização das unidades significantes. Os exercícios 7 e 8 foram elaborados de modo que não atendesse a nenhum critério de congruência semântica, tornandoos exercícios não-congruentes.

Este instrumento foi elaborado com o objetivo de propiciar a análise do conhecimento dos sujeitos da pesquisa referentes a conversão do registro em língua natural para as escrituras algébricas, em atividades que não possuem em seu enunciado um contexto pré-estabelecido. Sobretudo, analisar a capacidade dos estudantes em identificar e converter as unidades significantes em diferentes níveis de congruência semântica para a linguagem matemática.

Dessa forma, este instrumento de pesquisa foi aplicado a todos os acadêmicos, do primeiro ao quarto ano, de um curso de graduação em matemática de uma Universidade pública. Contudo, como o curso oferta tanto o grau em bacharel como o grau em licenciatura, optamos por selecionar apenas os acadêmicos de licenciatura, visto que eles possivelmente serão os 
futuros professores da Educação Básica e que lecionaram o conceito de inequação do $1^{\circ}$ grau com uma variável.

Deste modo, optado pelos acadêmicos da licenciatura, restringimos o número de acadêmicos por ano a quatro acadêmicos, visto que determinados anos acadêmicos possuíam apenas quatro acadêmicos que optaram pela licenciatura, dessa forma foi padronizado o número de quatro acadêmicos para cada ano do curso

Para o terceiro e quarto anos do curso que possuíam maior número de estudantes, estabelecemos como critério ordenar as fichas em ordem alfabética, e na sequência selecionar as fichas referentes ao números 1, 3, 5, 7 para o terceiro ano e as fichas 1, 4, 7, 10 para os acadêmicos do $4^{\circ}$ ano, para assim diversificar na seleção dos acadêmicos.

Para manter o anonimato dos acadêmicos, atribui-se a letra A maiúscula seguida por um número para representar o termo acadêmico e o número que o representa, além de um número ordinal seguido pela letra A maiúscula para representar o ano em que o acadêmico está. Por exemplo, o acadêmico A3 $-1^{\circ} \mathrm{A}$ se lê acadêmico três do primeiro ano.

Para analisar as resoluções dos acadêmicos, os procedimentos consistiram inicialmente na verificação das resoluções dos sujeitos da pesquisa referente a cada exercício, separando-os em acertos e erros, e a partir das resoluções incorretas, analisavam-se as conversões dos registros, em especial, as unidades significantes identificadas e convertidas, bem como o tratamento realizado para determinar a solução do exercício.

Sobretudo, nos pautamos em Damm (2015), no qual ela faz a seguinte indagação: "Como fazer uma análise cognitiva, em termos de registros, de atividades matemáticas?" (DAMM", 2015, p. 186). Neste caso, Damm elenca algumas etapas a serem realizadas:

Inicialmente devemos ter clara a distinção fundamental entre - tratamento: transformação de uma representação ficando no interior de um mesmo registro - conversão: transformação de uma representação mudando de registro. Em segundo lugar, as consequências dessa distinção seriam: a necessidade de verificar os tratamentos específicos a cada registro sem misturar com os tratamentos em outro registro [...]; o estudo de graus de congruência ou não congruência durante a conversão das representações. Aqui apareceriam duas questões-chave para a aprendizagem: a discriminação das unidades significantes em uma representação; o fechamento dos registros e a articulação entre registros (DAMM, 2015, p. 186-187).

Deste modo, apresenta-se na seção a seguir as análises e discussão dos dados obtidos evidenciando os principais erros e dificuldades identificadas nas resoluções dos acadêmicos sujeitos da pesquisa. 


\section{Análise e discussão dos dados}

Para organização dos acertos e erros, elaboramos um quadro contento todos os acadêmicos sujeitos da pesquisa separados por ano acadêmico e os oito exercícios no qual compõe o instrumento diagnóstico. Este quadro possibilita um panorama geral dos dados obtidos neste instrumento, cujo símbolo " $\checkmark$ " indica que a atividade foi resolvida corretamente, atendendo assim todos os critérios do exercício. O símbolo " $X$ " indica que houve algum erro em determinada passagem ou resposta final do exercício, já o símbolo "—" indica que o acadêmico não resolveu o exercício proposto.

\begin{tabular}{|c|c|c|c|c|c|c|c|c|c|}
\hline \multicolumn{10}{|c|}{ Instrumento de pesquisa -1} \\
\hline \multirow{2}{*}{ Ano acadêmico } & \multirow{2}{*}{ Acadêmicos } & \multicolumn{8}{|c|}{ Exercícios } \\
\hline & & 1 & 2 & 3 & 4 & 5 & 6 & 7 & 8 \\
\hline \multirow{4}{*}{$1^{\circ}$ ano } & $\mathrm{A} 1-1^{\circ} \mathrm{A}$ & $\checkmark$ & $\checkmark$ & $x$ & $\mathrm{x}$ & $\checkmark$ & $\mathrm{x}$ & $\mathrm{x}$ & $x$ \\
\hline & $\mathrm{A} 2-1^{\circ} \mathrm{A}$ & $\mathrm{x}$ & $x$ & $\mathrm{x}$ & $\mathrm{x}$ & $\checkmark$ & 一 & $x$ & - \\
\hline & $\mathrm{A} 3-1^{\circ} \mathrm{A}$ & $x$ & $x$ & $x$ & $x$ & $x$ & $\checkmark$ & - & $\checkmark$ \\
\hline & $\mathrm{A} 4-1^{\circ} \mathrm{A}$ & $\checkmark$ & $\checkmark$ & $\checkmark$ & $x$ & $\checkmark$ & $\checkmark$ & $x$ & $\checkmark$ \\
\hline & & & & & & & & & \\
\hline \multirow{4}{*}{$2^{\circ}$ ano } & $\mathrm{A} 1-2^{\circ} \mathrm{A}$ & $\checkmark$ & $\checkmark$ & $\checkmark$ & $x$ & $\checkmark$ & $\checkmark$ & $x$ & $\sqrt{ }$ \\
\hline & $\mathrm{A} 2-2^{\circ} \mathrm{A}$ & $x$ & $x$ & $x$ & $x$ & $x$ & $x$ & $x$ & $x$ \\
\hline & $\mathrm{A} 3-2^{\circ} \mathrm{A}$ & $\checkmark$ & $x$ & $x$ & $x$ & $\checkmark$ & $x$ & $x$ & $x$ \\
\hline & $\mathrm{A} 4-2^{\circ} \mathrm{A}$ & $\checkmark$ & $\checkmark$ & $x$ & $x$ & $\checkmark$ & $\checkmark$ & $x$ & $x$ \\
\hline \multirow{4}{*}{$3^{\circ}$ ano } & $\mathrm{A} 1-3^{\circ} \mathrm{A}$ & $\checkmark$ & $\checkmark$ & $\checkmark$ & $x$ & $x$ & $\checkmark$ & $x$ & $\checkmark$ \\
\hline & $\mathrm{A} 2-3^{\circ} \mathrm{A}$ & $\checkmark$ & $x$ & $x$ & $x$ & $x$ & $x$ & $x$ & $x$ \\
\hline & $\mathrm{A} 3-3^{\circ} \mathrm{A}$ & $\checkmark$ & $x$ & $\checkmark$ & $x$ & $x$ & $x$ & $x$ & $x$ \\
\hline & $\mathrm{A} 4-3^{\circ} \mathrm{A}$ & $\checkmark$ & $x$ & $x$ & $x$ & $x$ & $x$ & $x$ & $x$ \\
\hline \multirow{4}{*}{$4^{\circ}$ ano } & $\mathrm{A} 1-4^{\circ} \mathrm{A}$ & $\checkmark$ & $x$ & $x$ & $x$ & $\checkmark$ & $\checkmark$ & $\checkmark$ & $\sqrt{ }$ \\
\hline & $\mathrm{A} 2-4^{\circ} \mathrm{A}$ & $\checkmark$ & $x$ & $x$ & $x$ & $\checkmark$ & $x$ & $x$ & $x$ \\
\hline & $\mathrm{A} 3-4^{\circ} \mathrm{A}$ & $\checkmark$ & $\checkmark$ & $\checkmark$ & $x$ & $x$ & $\checkmark$ & $x$ & $\checkmark$ \\
\hline & $\mathrm{A} 4-4^{\circ} \mathrm{A}$ & $x$ & $x$ & $x$ & $x$ & - & - & - & - \\
\hline
\end{tabular}

Quadro 5 - Organização dos acertos, erros e as resoluções não realizadas identificadas nos quatro anos do curso referente aos oito exercício.

Fonte: autores.

Referente ao exercício 1, no qual atende aos três critérios de congruência semântica, 12 dos 16 acadêmicos resolveram o exercício corretamente, sendo que, neste caso, apenas os acadêmicos $\mathrm{A} 2-1^{\circ} \mathrm{A}, \mathrm{A} 3-1^{\circ} \mathrm{A}, \mathrm{A} 2-2^{\circ} \mathrm{A}$ e $\mathrm{A} 4-4^{\circ} \mathrm{A}$ erraram o exercício.

Dentre os erros identificados neste exercício, observamos que dois deles são referentes a conversão, no qual um está relacionado a conversão incorreta da unidade significante maior que, visto que no registro terminal o acadêmico converte para o símbolo de $\geq$ (maior/igual). Já para outro erro envolvendo conversão, o estudante sendo este do acadêmico 
A2 $-1^{\circ} \mathrm{A}$ não montou uma expressão algébrica para resolução do exercício, conforme é apresentado na figura 3 a seguir.

\begin{tabular}{|c|c|}
\hline Resolução & Resposta final \\
\hline $\begin{aligned} x=26 \quad & 26 \\
& +26 \\
& 26 \\
& \\
& \end{aligned}$ & $x=26$. \\
\hline
\end{tabular}

Figura 3 - Resolução incorreta do acadêmico A2 - $1^{\circ} \mathrm{A}$ - exercício 1.

Fonte: autores.

Observe que o estudante não conseguiu realizar a conversão, atribuindo um valor fixo qualquer a variável x e somando com duas vezes o seu próprio valor, obtendo o resultado de 78, que de fato é um resultado válido, porém, parcial, uma vez que a variável x pode assumir infinitos valores de acordo com as condições especificadas no enunciado.

Deste modo, pressupõe-se que o acadêmico não compreendeu o que se pedia no exercício, sobretudo, a unidade significante maior foi interpretada apenas como uma condição para o resultado final, que neste caso teria que ser maior que 27 , e não como uma unidade significante do conceito de inequação.

Já as demais resoluções erradas referentes ao exercício 1 foram oriundas de tratamentos algébricos incorretos.

Com relação ao exercício 2, cujo nível de congruência é o 3, ou seja, congruente, percebe-se que o número de acadêmicos que erraram o exercício foi superior ao exercício 1 , sendo um total 10 dos 16 acadêmicos.

Um dos principais erros identificados referente a conversão está relacionado ao fato de os acadêmicos não converterem o sinal negativo que acompanha o número três no enunciado, cujo exemplo pode ser observado na figura 4 a seguir.

\begin{tabular}{|c|c|}
\hline \multicolumn{1}{|c|}{ Resolução } & Resposta final \\
\hline $3 x>14$ & $x>\frac{14}{3}$ \\
$x>\frac{14}{3}$ & \\
\hline
\end{tabular}

Figura 4 - Resolução do acadêmico $\mathrm{A} 3-3^{\circ} \mathrm{A}-$ exercício 2. Fonte: autores.

Este erro foi identificado em três das 10 resoluções incorretas, possivelmente derivados 
da má interpretação do sinal, ou até mesmo, falta de atenção visto que a unidade significante neste caso não precisava de ser convertida, já que estava no registro algébrico.

Um erro incomum para este exercício foi identificado na resolução do acadêmico A3 $1^{\circ} \mathrm{A}$ na qual o estudante realiza todos os procedimentos de tratamento corretamente. Contudo, na conversão do registro em língua natural para as escrituras algébricas, montou um inequação contendo o sinal de $\geq$ (maior/igual) enquanto que no enunciado a unidade significante está explícita em língua corrente: maior. Erro este possivelmente executado devido à não atenção as unidades significantes do exercício.

Referente ao tratamento, o principal erro presente neste exercício 2 é o erro envolvendo uma das propriedades de operações de inequação, ou seja, os acadêmicos multiplicam/dividem por um valor negativo ambos os lados da inequação, porém, não invertem o sinal de desigualdade. Este erro foi identificado em três das 10 resoluções errôneas. Os demais erros são referentes a descuidos no tratamento algébrico ou na apresentação da solução final.

Para o exercício 3, cujo enunciado atende a dois critérios de congruência semântica, o índice de erros foi maior que os dois primeiros exercícios, totalizando 11 das 16 resoluções analisadas.

Neste exercício, as condições de correspondência semântica e univocidade semântica terminal são satisfeitas. No entanto, a ordem das unidades significantes presentes no registro em língua natural não coincidem com a mesma ordem das unidades significantes fixadas na conversão para o registro algébrico. As unidades para este exercício 3 são: não é igual nem maior; 12; diferença entre $x$ e seu triplo.

Dentre os erros identificados nesse exercício, três são relacionados a conversão do registro em língua natural para as escrituras algébricas. Destes três erros, duas resoluções, sendo estas dos acadêmicos A2 $-1^{\circ} \mathrm{A}$ e A4 $-3^{\circ} \mathrm{A}$ apresentaram expressões que não atendem as condições estabelecidas no enunciado, já a resolução do acadêmico A1 - $1^{\circ} \mathrm{A}$ apesar de ter identificado e convertido todas as unidades significantes presentes no enunciado, montou uma expressão incorreta referente a unidade significante a diferença entre $x$ e seu triplo, conforme pode ser visualizada na figura 5 a seguir.

\begin{tabular}{|c|c|}
\hline Resolução & Resposta final \\
\hline $3 x-x<12$ & $x<6$ \\
$2 x<12$ & \\
$x<6$ & \\
\hline
\end{tabular}


Figura 5 - Resolução incorreta no acadêmico A1 - $1^{\circ} \mathrm{A}$ - exercício 3.

Fonte: autores.

Este fato possibilita inferir que o acadêmico possui dificuldades em converter a unidade significante corretamente devido ao fato de obter como resultado uma diferença de termos que resultará em um valor negativo para a variável. É mais fácil supor que a variável sempre será positiva do que negativa.

Já os demais erros identificados neste exercício estão relacionados aos tratamentos realizados na expressão algébrica convertida. Neste caso, novamente destaca-se o erro referente a multiplicação/divisão por um valor negativo sem alterar o sinal de desigualdade, no qual três acadêmicos efetuaram essa operação incorretamente. Contudo, identificamos também dois erros relacionados a operações de divisão, já os demais referem-se a procedimentos como trocar o sinal de maior pelo sinal de igual no meio do procedimento de tratamento, ou até então, não realizar o tratamento, apenas a conversão para a expressão algébrica inicial.

Para o exercício 4, cuja nível de congruência semântica é o mesmo que o exercício 3, satisfazendo a dois dos três critérios de congruência semântica, nota-se que o número de erros foi o maior no instrumento de pesquisa, no qual todos os 16 acadêmicos efetuaram procedimentos incorretos em alguma etapa da resolução.

Neste exercício tanto a correspondência semântica como a ordem das unidades significantes são satisfeitas, porém, a univocidade semântica terminal não é, visto que a combinação das unidades significantes um número inteiro somado com seu igual possibilita diferentes interpretações como por exemplo, 1+1, 20+20, etc. O mesmo ocorre com a unidade significante não é menor, em razão de que não é menor pode implicar tanto na conversão para unidade significante $>$ (maior) e/ou $=$ (igual).

Contudo, este critério de congruência semântica não foi determinante nos erros identificados nas resoluções dos acadêmicos, mas sim as unidades significantes 2 multiplicado com o seu triplo, conforme exemplifica a figura 6 a seguir.

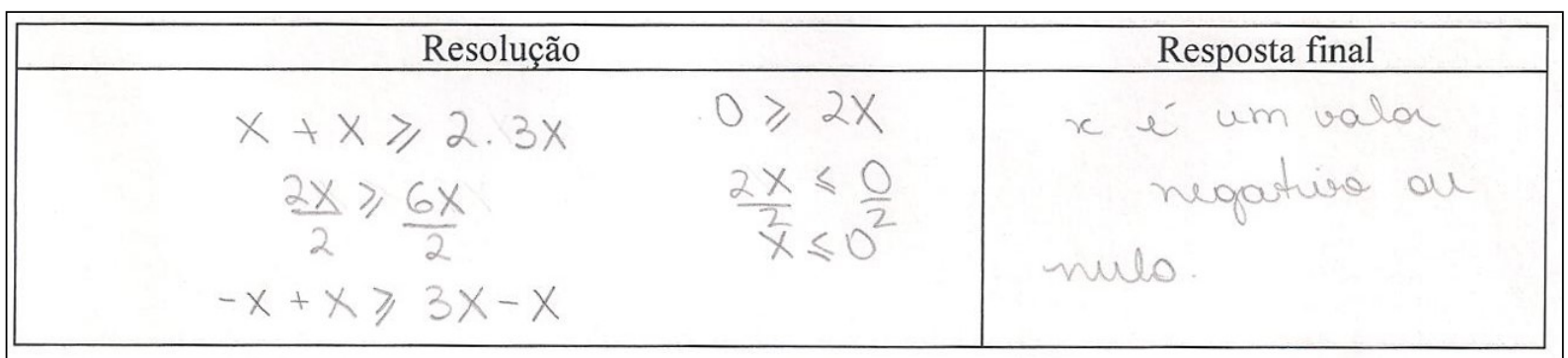

Figura 6 - Resolução incorreta do acadêmico A1 - $3^{\circ} \mathrm{A}$.

Fonte: autores.

Diante dos dados obtidos e analisados, observamos que 13 dos 16 acadêmicos efetuaram 
exatamente a mesma conversão como apresentada na resolução da figura 6 . Ou seja, os acadêmicos interpretaram as unidades significantes 2 multiplicado com seu triplo com sendo a multiplicação de 2 com o triplo da variável x e não como sendo 2 multiplicado com seu triplo (6). Os outros três erros restantes foram semelhantes a este, apenas alterando a representação algébrica em alguns aspectos, como por exemplo3 $(x+x) \geq 2$.

O exercício 5 é o primeiro do instrumento a ser resolvido cuja atividade de conversão satisfaz apenas um dos critérios de congruência semântica.

Neste exercício, foram identificadas sete resoluções incorretas e uma não realizada. Número inferior se comparada aos exercícios 3 e 4 que continham um critério de congruência semântica a mais em suas elaborações.

Fazendo uma leitura do enunciado do exercício, identifica-se as seguintes unidades significantes: mínimo; 17; soma; número par; sucessor.

No que se refere aos erros, quatro dos sete erros estão relacionados a utilização do sinal de igual (=) para representar uma expressão algébrica que atendesse as informações fornecidas no enunciado, conforme apresenta-se na figura 7 a seguir.

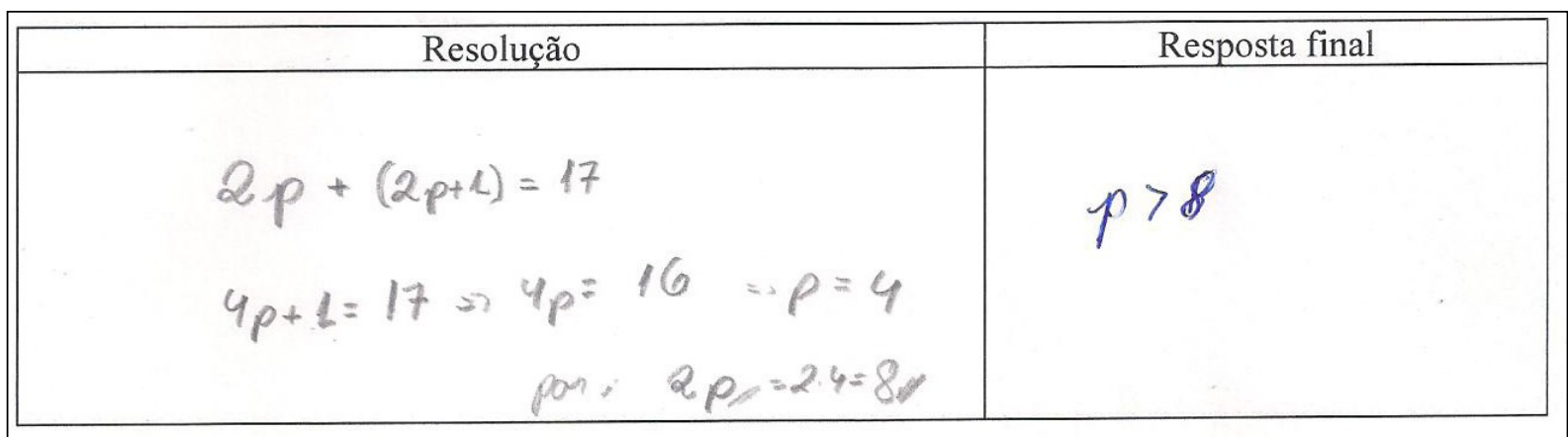

Figura 7 - Resolução do acadêmico A4 - $3^{\circ} \mathrm{A}$ - exercício 5.

Fonte: autores.

Nota-se por meio deste erro que alguns dos acadêmicos não identificaram a unidade significante mínimo como sendo um termo referente ao conceito de inequação, influenciando assim para que alguns acadêmicos pensassem que o exercício se tratava de uma equação.

Outras duas situações que nos chamaram a atenção, corresponde as resoluções dos acadêmicos $\mathrm{A} 2-3^{\circ} \mathrm{A}$ e $\mathrm{A} 3-4^{\circ} \mathrm{A}$. Para realizar o tratamento algébrica, ambos os acadêmicos montaram uma expressão do tipo $x+x+1 \geq 17$, na qual representaram a unidade significante número par como sendox e consequentemente a unidade significante sucessor, $\operatorname{como} x+1$. Estas representações nos fizeram inferir que ambos os acadêmicos identificaram as unidades significantes como sendo genéricas, o que está correto, contudo, consideraram x como sendo par, mas que neste contexto se tornou incorreto, uma vez que considerar x como sendo par está correto, porém, representar x como sendo par não está correto. Para que a representação seja 
válida é necessário que o número 2 preceda a variável x.

As demais resoluções apresentaram erros na conversão, especificamente na expressão inicial referente a combinação das unidades significantes número par e seu sucessor.

Referente ao exercício 6, este foi elaborado de modo que atendesse apenas a um critério de congruência semântica, e que fosse diferente do critério que o exercício 5 possui. Deste modo, decidimos por elaborar um exercício no qual atendesse apenas a unidade significante, neste caso o critério 3, ou seja, a ordem das unidades significantes. Assim sendo, temos como unidades significantes para este exercício os termos: $x$; diferença; dobro e número número não negativo.

Neste exercício 6, o número de resoluções inválidas foram próximas ao exercício 5 , neste caso foram sete resoluções incorretas e duas resoluções não feitas.

Das sete resoluções incorretas, observamos que todas elas apresentavam erros relacionados a conversão do registro em língua natural para registro algébrico (registro maior sistemas de escrita). Dentre estes erros envolvendo conversões, analisamos quais unidades significantes os acadêmicos possuíram maior dificuldade em identificar e converter para as escrituras algébricas, visto que o critério de univocidade semântica terminal e o critério de correspondência semântica entre as unidades significantes, são critérios que envolvem tanto o conhecimento semântico como o conhecimento lexical dos acadêmicos.

Analisado as resoluções dos acadêmicos, notamos que referente a unidade significante número não negativo, das sete resoluções incorretas, seis não conseguiram representar a unidade corretamente em sua forma algébrica. Este fato pode estar ligado ao critério de univocidade semântica, uma vez que o termo número não negativo possibilita ao estudante interpretar como sendo qualquer número maior que zero, excluindo assim o zero dos valores restrito, porém, como o zero não é um número positivo nem negativo, inclui-se ele nos valores referentes a unidade significante número não negativo.

A figura 8 a seguir apresenta um exemplo de resolução envolvendo tal erro.

\begin{tabular}{|c|l|}
\hline \multicolumn{1}{|c|}{ Resolução } & \multicolumn{1}{c|}{ Resposta final } \\
\hline$x-2 X>0$ & pora $x$ minores \\
$\Rightarrow-X>0$ & que zro \\
$\Rightarrow X<0$ & $x \in \mathbb{R}^{-}$ \\
\hline
\end{tabular}

Figura 8 - Resolução incorreta do acadêmico A3 - $2^{\circ} \mathrm{A}$ - exercício 6.

Fonte: autores. 
Como observa-se na figura 8, o acadêmico até escreve em língua natural a resposta final especificando-a, contudo, incorreta pelo fato de não abordar o zero em tal inequação.

Além destes erros, identificamos também que o termo resulta presente no enunciando do exercício pode ter influenciado nas respostas de dois dos sete acadêmicos. Ao analisar as respostas, identificamos que os acadêmicos $\mathrm{A} 2-3^{\circ} \mathrm{A}$ e $\mathrm{A} 2-4^{\circ} \mathrm{A}$ montaram uma equação algébrica para resolver o exercício. Este fato possibilita inferior que o critério de correspondência semântica pode ter influenciado em tais respostas. Haja visto que o termo resulta é uma unidade significante do conceito de equação, que por sua vez atrelado ao fato de não ter uma unidade significante que associe ao sinal de desigualdade da inequação, possa ter confundi-los na hora da conversão. Já o acadêmico A1 - $1^{\circ} \mathrm{A}$, assim como o exercício 3, interpretou Determine $x$ de modo que ao realizar a diferença com seu dobro como sendo a diferença do dobro de $x$ e $x$, ou seja, escreveu algebricamente a inequação $2 x-x \geq 0$ quando o correto seria $x-2 x \geq 0$.

O exercício 7, juntamente com o exercício 8 teoricamente são os mais difíceis deste instrumento de pesquisa uma vez que nenhum dos critérios de congruência semântica são atendidos em seus enunciados, tornando-os exercícios não-congruentes. Deste modo, temos como unidades significantes para este exercício: valor máximo; produto; 3 e dobro.

No que se refere ao exercício 7, nota-se que dos 16 acadêmicos sujeitos da pesquisa, apenas um acadêmico realizou o exercício corretamente, 13 erraram em algum procedimento da resolução e dois não resolveram o exercício.

No que se refere aos erros dos acadêmicos, observamos que dos 13 erros identificados, 11 estão relacionados a combinação das unidades significantes: o produto entre 3 e seu dobro. Este fato pode ser verificado em uma das resoluções dos acadêmicos, conforme é apresentado na figura 9 a seguir.

\begin{tabular}{|c|c|}
\hline \multicolumn{1}{|c|}{ Resolução } & Resposta final \\
\hline$x \leqslant 3.2 x$ & $x \geqslant 0$ \\
$x \leqslant 6 x$ & \\
$-5 x \leqslant 0$ & \\
$x \geqslant 0$ & \\
\hline
\end{tabular}

Figura 9 - Resolução incorreta do acadêmico A1 - $2^{\circ} \mathrm{A}$ - exercício 7. Fonte: autores.

Nota-se que este erro é semelhante ao erro identificado no exercício 4, visto que as 
unidades significantes que ocasionaram o erro são semelhantes. Este erro é oriundo da interpretação equivocada ao supor que os termos seu dobro referia-se a variável x, o que na verdade refere-se o termo que precede a unidade significante, ou seja, o número 3.

Dos dois erros restantes, um refere-se a conversão da expressão algébrica inicial, visto que o acadêmico apenas apresentou um tipo de solução para o exercício (um número qualquer para a variável x), na qual utilizou-se de igualdade. Já o segundo erro refere-se ao tratamento. Neste caso o acadêmico realizou corretamente a conversão para o registro simbólico algébrico, entretanto, ao realizar o tratamento efetuou incorretamente uma operação de multiplicação.

Para o exercício 8, cujo nível de congruência semântica é igual ao exercício 7, ou seja, não congruente, nota-se que os erros foram inferiores ao número de erros identificados no exercício 7.

Este exercício possui como unidades significantes os seguintes termos: limite inferior; número $x$; somado; 8; diferença; dois números iguais.

Nota-se a complexidade em compreender o enunciado do exercício, bem como interpretar as unidades significantes presentes, visto que há diversos aspectos que podem influenciar em uma resposta incorreta neste exercício, tais como a ordem das unidades significantes, os termos sinônimos, etc.

Dentre essas unidades significantes, observamos que a unidade significante limite inferior influenciou nas respostas incorretas de dois acadêmicos. Esta unidade significante faz referência ao símbolo $\geq$ (maior/igual) neste contexto, e os acadêmicos A1 $-1^{\circ} \mathrm{A}$ e A2 $-4^{\circ} \mathrm{A}$ interpretaram como sendo $>$ (maior) que dois números iguais. Contudo, além deste fato, os cinco erros restantes identificados neste exercício refere-se também ao sinal de desigualdade da inequação. Neste caso os demais acadêmicos consideram a expressão algébrica como sendo uma equação, no qual podemos inferir que estes erros justifica-se pela "combinação" da ausência dos três critérios de congruência semântica.

Observe que a unidade significante limite inferior dos valores além de não atender a ordem das unidades significantes, é prejudicado em sua interpretação quando lê o restante do enunciado: ...que um número x somado com 8 pode assumir é "igual" a diferença de dois números iguais. O termo igual é uma unidade significante do conceito de equação, mas que neste caso não apresenta correspondência semântica visto que o termo igual refere-se aos valores que o segundo membro da inequação pode assumir. Está complexidade de interpretação levou os acadêmicos a confundirem o exercício de inequação como sendo um exercício de equação. 
Realizando um tratamento no enunciado em língua natural do exercício 8, podemos obter a seguinte questão: sabe-se que os valores que um número $x$ somado com 8 pode assumir, é igual ao limite inferior da diferença de dois números iguais.

Note que ao alterar a ordem das unidades significantes do enunciado, pode tornar sua compreensão "menos complexa" quando comparado com o enunciado original do exercício. Neste exemplo de tratamento, o termo igual não faz referência apenas a diferença de dois números iguais, mas sim ao limite inferior da diferença de dois números iguais, referenciando assim não apenas o segundo membro da inequação, mas também o sinal de desigualdade que divide a inequação em dois membros, propiciando a identificação da relação de desigualdade existente.

O quadro 6 a seguir apresenta a quantidade numérica de acertos, erros e os exercícios não resolvidos deste instrumento de pesquisa referente as resoluções dos 16 acadêmicos sujeitos deste estudo, categorizados pelo ano da graduação.

\begin{tabular}{|c|c|c|c|}
\hline \multicolumn{4}{|c|}{ Instrumento de pesquisa - 1 } \\
\hline Ano acadêmico & Acertos & Erros & Não resolvidos \\
\hline $1^{\mathrm{o}}$ ano & 12 & 17 & 3 \\
\hline $2^{\mathrm{o}}$ ano & 12 & 20 & 0 \\
\hline $3^{\mathrm{o}}$ ano & 9 & 23 & 0 \\
\hline $4^{\mathrm{o}}$ ano & 12 & 16 & 4 \\
\hline
\end{tabular}

Quadro 6 - Total numérico de erros, acertos e exercícios não resolvidos. Fonte: autor.

Considerando que o instrumento de pesquisa é composto por oito exercícios, e que foram abordados como sujeitos de pesquisa quatro alunos de cada ano da graduação, temos um total de 32 resoluções para cada ano acadêmico referente a este instrumento diagnóstico.

Deste modo, nota-se que o número de acertos foram menores que o número de erros, ou seja, mais erros do que acertos.

A princípio, esperava-se que os acadêmicos do $4^{\circ}$ ano obtivessem um desempenho maior comparado aos acadêmicos dos primeiros anos, em razão da experiência e habilidade com operações em conceitos básicos, sobretudo, advindos da prática do estágio curricular, disciplina na qual o $3^{\circ}$ e $4^{\circ}$ anos da graduação possuem. Entretanto, observa-se que o número de acertos foram iguais para o $1^{\circ}, 2^{\circ}$ e $4^{\circ}$ anos do curso de Licenciatura em Matemática, atingindo um total percentual de $40 \%$ de acertos. Já para o $3^{\circ}$ ano do curso, este número foi ainda menor, obtendo nove acertos de 32 resoluções. Um total percentual de $30 \%$ de acertos.

Notamos ainda que referente as unidades significantes, aquelas cuja interpretação possam ser ambíguas, aspectos estes relacionados a não univocidade semântica terminal, o 
número de erros foram superiores quando comparados com as demais unidades significantes, sobretudo, quando "combinadas" com a ausência dos demais critérios de congruência semântica.

\section{Considerações finais}

Neste artigo, buscamos realizar análise do desempenho de acadêmicos dos quatro anos de um curso de Licenciatura em Matemática em atividades envolvendo o conceito de inequação em seus diferentes níveis de congruência semântica.

Neste estudo, termos do tipo maior que, menor que, não é maior, não é menor, no mínimo, no máximo etc., são condições que possam representar uma desigualdade de valores, pressupondo assim a utilização do conceito de inequação para sua resolução. Ou seja, identificar, interpretar e converter esses termos para a representação algébrica é parte essencial no processo de resolução de atividades envolvendo o conceito de inequação.

Podemos inferir que alguns exercícios tais como o exercício 4 e o exercício 7 cuja relação sintática é mais "ambígua" influenciou na conversão incorreta dos acadêmicos. Este tipo de situação nos faz refletir sobre a sintática dos enunciados bem como a influência e importância do conhecimento linguístico em exercícios e problemas matemáticos.

Outro fator que contribuiu para os erros dos acadêmicos está relacionado a atenção dos estudantes com o enunciado do exercício, visto que em muitos casos as unidades significantes são convertidas corretamente, porém, erros no tratamento algébrico envolvendo operações matemáticas simples invalidam suas repostas

Contudo, cabe mencionar que algumas dificuldades aqui identificadas apresentam semelhanças com outras pesquisas já realizadas envolvendo o conceito de inequação. Erros relacionados a multiplicação/divisão por um valor negativo, onde não altera-se o sinal de desigualdade foram destaques em pesquisas como a de Campos e Giusti (2008) e Travassos e Rezende (2017), sobretudo, erros envolvendo a utilização de inequações baseadas no conceito de equação também foram mencionadas na pesquisa de Campos e Giusti (2008).

Esta pesquisa apresentou de modo geral, dados preocupantes, visto que o conceito de inequação do $1^{\circ}$ grau com uma variável é um conteúdo do Ensino Fundamental e Médio, mas que como apresentamos, graduandos do $4^{\circ}$ ano do curso apresentaram erros, inclusive em exercícios congruentes. 


\section{Referências}

BRASIL. Ministério da Educação. Secretaria de Educação Fundamental. Parâmetros Curriculares Nacionais: Matemática. ( $3^{\circ}$ e $4^{\circ}$ ciclos do ensino fundamental). Brasília: MEC, 1998.

BRASIL. Ministério da Educação. Secretaria de Educação Média e Tecnológica. Parâmetros Curriculares Nacionais (Ensino Médio). Brasília: MEC, 2000.

BRASIL. Ministério da Educação. Base Nacional Comum Curricular. $3^{\mathrm{a}}$ ed. Brasília: MEC, 2017. Disponível em: $<\mathrm{http}$ ://basenacionalcomum.mec.gov.br/images/BNCC_20dez_site.pdf $>$. Acesso em: 19 jan. 2018.

CAMPOS, Tânia M. M.; GIUSTI, V. H. Resolução de Desigualdades com uma Incógnita: uma análise de erro. Unión (San Cristobal de La Laguna), v. 14, p. 37-48, 2008.

DAMM, R.F. Registros de Representação. In: MACHADO, S.D.A.(org). Educação Matemática: Uma (nova) introdução. São Paulo: Educ, 2015, p.167-188.

DUVAL, R. Registros de representações semióticas e funcionamento cognitivo da compreensão em Matemática. In: Aprendizagem em Matemática. Machado, S. D. A. (org.). pp. 11-33. Campinas, SP: Papirus, 2003.

DUVAL, R. Semiósis e Pensamento Humano: Registros semióticos e aprendizagens intelectuais (Fascículo I). Tradução: Lênio Fernandes Levy e Marisa Rosâni Abreu da Silveira. São Paulo: Editora Livraria da Física, 2009.

DUVAL, R.; FREITAS, J. L. M.; REZENDE, V. Entrevista: Raymond Duval e a Teoria dos Registros de Representação Semiótica. Revista Paranaense de Educação Matemática, v. 2, p. 10-34, 2013.

GIL, A. C. Métodos e Técnicas de Pesquisa Social. São Paulo: Atlas, 2008.

MAGALHÃES, A. F. Estudos das inequações: contribuições para a formação do professor de matemática na licenciatura. 2013. 127 f. Dissertação (Mestrado em Educação Matemática) Universidade Federal de Ouro Preto, Ouro Preto, 2013.

MELO, M. O ensino de desigualdades e inequações em um curso de Licenciatura em

Matemática. 2007. 81 f. Dissertação (Mestrado em Educação) - Pontifícia Universidade Católica de São Paulo, São Paulo, 2007.

SOUZA, Vera Helena Giusti. O uso de vários registros na resolução de inequações: uma abordagem funcional gráfica. 2008. 292 f. Tese (Doutorado em Educação Matemática) - Pontifícia Universidade Católica de São Paulo, São Paulo, 2008.

TRAVASSOS, W. B.; REZENDE, V. O Software Aplusix e a Resolução de Inequações: um estudo de erros e acertos de estudantes do $1^{\circ}$ ano de Matemática. Educação Matemática em Revista, v. 22, p. 85-98, 2017. 